\title{
Longitudinal injection transients in an electron storage ring
}

\author{
J. M. Byrd ${ }^{1,2}$ and S. De Santis ${ }^{1}$ \\ ${ }^{1}$ Lawrence Berkeley National Laboratory, One Cyclotron Road, Berkeley, California 94720 \\ ${ }^{2}$ Department of Physics, University of California, Davis, Davis, California 95616
}

(Received 2 November 2000; published 9 February 2001)

\begin{abstract}
We present the results of an experimental study of the longitudinal beam dynamics at injection in the advanced light source, an electron storage ring. By measuring the longitudinal bunch distribution following injection using a streak camera, we were able to study several useful and interesting effects as well as improve overall injection efficiency. These include measurement and correction of the phase and energy offsets at injection, measurement of the injected bunch length and energy spread, direct observation of phase space filamentation due to the spread in synchrotron frequencies, and measurement of the effective damping rate of the bunch shape including radiation damping and decoherence. We have also made some initial studies of the decay of an uncaptured beam at injection which may provide a novel means of measuring the radiation loss per turn.
\end{abstract}

DOI: 10.1103/PhysRevSTAB.4.024401

PACS numbers: 41.85.-p, 41.75.Ht, 29.27.Fh, 29.27.Bd

\section{INTRODUCTION}

Although injection is a crucial process in any storage ring, the details of the longitudinal beam dynamics at injection in electron storage rings are usually ignored as long as there is adequate capture of the injected beam. This is because the radiation damping eventually damps the injected beam within a fraction of a second to the natural storage ring bunch length and energy spread, a time much shorter than the typical storage time of the beam. There are two notable exceptions where the injection transients are important: damping rings for linear colliders and continuous top-up injection in synchrotron light sources. In a linear collider, the electron (or positron) beam is transferred from the linac to the damping ring sometime during its acceleration, where its longitudinal and transverse emittances are damped. The beam is subsequently reinjected into the linac and accelerated to the collision point. The performance of the collider is determined, in part, by the time necessary for the beam to stay in the damping ring and reach the appropriate emittance. Thus it is essential that the transient response of the injected beam be understood and controlled. In continuous top-up mode at synchrotron light sources, a small amount of beam is injected with the stored beam keeping the average beam current practically constant. In this case, the injection transients must be controlled such that the average energy spread of the beam is not increased and that off energy electrons are not lost, creating radiation problems. Furthermore, the study of beam transients, whether from injection or from kicking a stored beam, provides general insight into the dynamics of the beam.

We present in this paper an experimental study of injection transients in longitudinal phase space at the advanced light source (ALS), a $1.5 \mathrm{GeV}$ electron storage ring optimized for producing high brightness synchrotron radiation. Machine parameters relevant to our experiments are listed in Table I. Using a dual-scan synchroscan streak camera (SC), we have recorded the longitudinal bunch distribution as a function of time in the storage ring following injection. With the streak camera, we can measure the injected bunch length and energy spread as well as identify injection phase and energy offsets. We can also directly observe the transient due to a longitudinal phase mismatch of the injected bunch and the subsequent phase filamentation through the spread in synchrotron frequencies within the bunch induced by the nonlinear rf voltage. In addition to these studies of a stored beam, we have also recorded the injection transient of an uncaptured beam (i.e., no rf voltage) and have used this as a novel technique for measuring the radiation loss per turn.

There have been considerable analytical and experimental studies of the transients of kicked beams [1-10]. However, most of the experimental studies to date have been for betatron oscillations. The experimental studies of longitudinal transients in the Stanford Linear Collider (SLC) damping rings $[9,10]$ were done before the availability of dual-sweep streak cameras and thus were not able to

TABLE I. Nominal ALS parameters.

\begin{tabular}{clc}
\hline \hline Parameter & \multicolumn{1}{c}{ Description } & Value \\
\hline$E$ & Beam energy & $1.5 \mathrm{GeV}$ \\
$C$ & Circumference & $196.8 \mathrm{~m}$ \\
$h$ & Harmonic number & 328 \\
$f_{\mathrm{rf}}$ & rf frequency & $500 \mathrm{MHz}$ \\
$f_{0}$ & Revolution frequency & $1.523 \mathrm{MHz}$ \\
$V_{\mathrm{rf}}$ & rf voltage & $1.1 \mathrm{MV}$ \\
$Q_{s}$ & Synchrotron tune & 0.0075 \\
$U_{0}$ & Radiation loss/turn & $92 \mathrm{keV}$ \\
$\alpha$ & Momentum compaction & $1.62 \times 10^{-3}$ \\
$\tau$ & Long. rad. damping time & $13.5 \mathrm{~ms}$ \\
$\sigma_{z \infty}$ & rms natural bunch length & $4.3 \mathrm{~mm}$ \\
$\sigma_{\epsilon \infty}$ & rms $\delta E / E$ & $6.5 \times 10^{-4}$ \\
\hline \hline
\end{tabular}


record a single injection transient over an appreciable period of time. Moshammer [6-8] studied the injection process both theoretically and via computer simulation and developed several useful expressions for the time evolution of the bunch moments with arbitrary injection conditions in the case of a quadratic tune dependence on oscillation amplitude. This was applied to both betatron and synchrotron transients. We summarize this work and review its application to longitudinal transients in the ALS in Sec. II. The results of measurements of the longitudinal injection transients in the ALS using a dual-sweep synchroscan streak camera are described in Sec. III. Our conclusions are given in Sec. IV.

\section{INJECTION TRANSIENTS IN LONGITUDINAL PHASE SPACE}

Consider the phase space representation of the injection of a bunch in an rf bucket as illustrated in Fig. 1. Figure 1a shows a case where the bunch distribution is offset in phase from the bucket center and the bunch area is also mismatched to the stored bunch area. Following injection, the bunch centroid rotates about the center of the bucket at the synchrotron frequency. The bunch distribution also rotates about its center at the synchrotron frequency. The distribution appears, as shown, a quarter of a synchrotron period later. Figure $1 \mathrm{~b}$ shows the case where the same bunch is injected with an energy offset. Figure 1c shows a case where the bunch distribution is injected at the center of the bucket but the phase space area is still mismatched. In this case, the bunch distribution simply rotates about the center of the rf bucket. As in the case of the ALS booster and storage ring, Figs. 1a-1c show a situation where the injected energy spread is close to the natural energy spread and the injected bunch length is about 4 times the natural bunch length. We assume that the orientation of the injected bunch ellipse is along the phase axis as shown in the figure.

Following injection, the initial distribution will begin to filament due to the amplitude dependence of the synchrotron frequency in a time typically less than the radiation damping time. An example of the phase space filamentation made using a tracking simulation is shown for ALS parameters in Fig. 2. The distribution eventually damps to the natural storage ring distribution. Snapshots of the phase space distribution are shown over 20000 turns

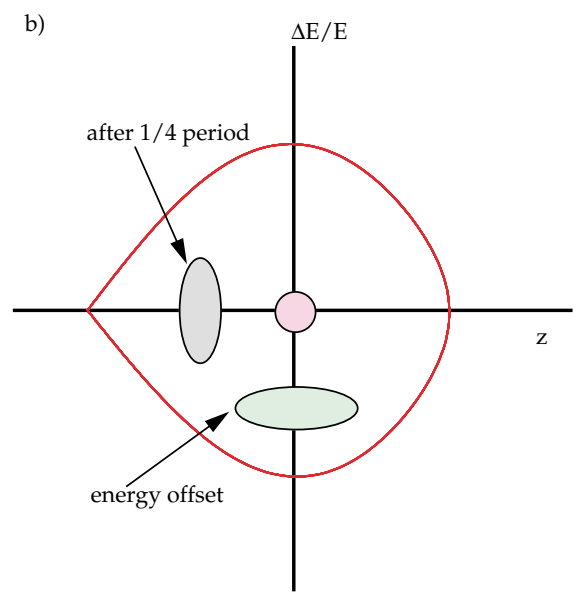

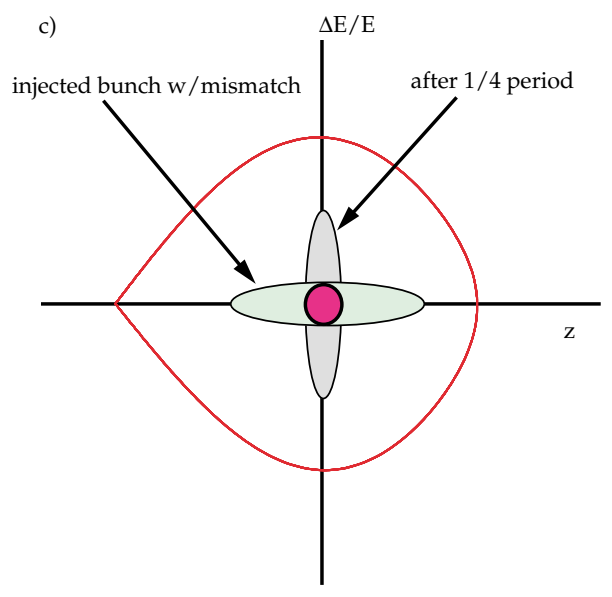

FIG. 1. (Color) Illustration of bunch oscillations at injection for the ALS. (a) Phase offset along with phase space mismatch. (b) Energy offset with phase space mismatch. (c) Phase space mismatch only. 


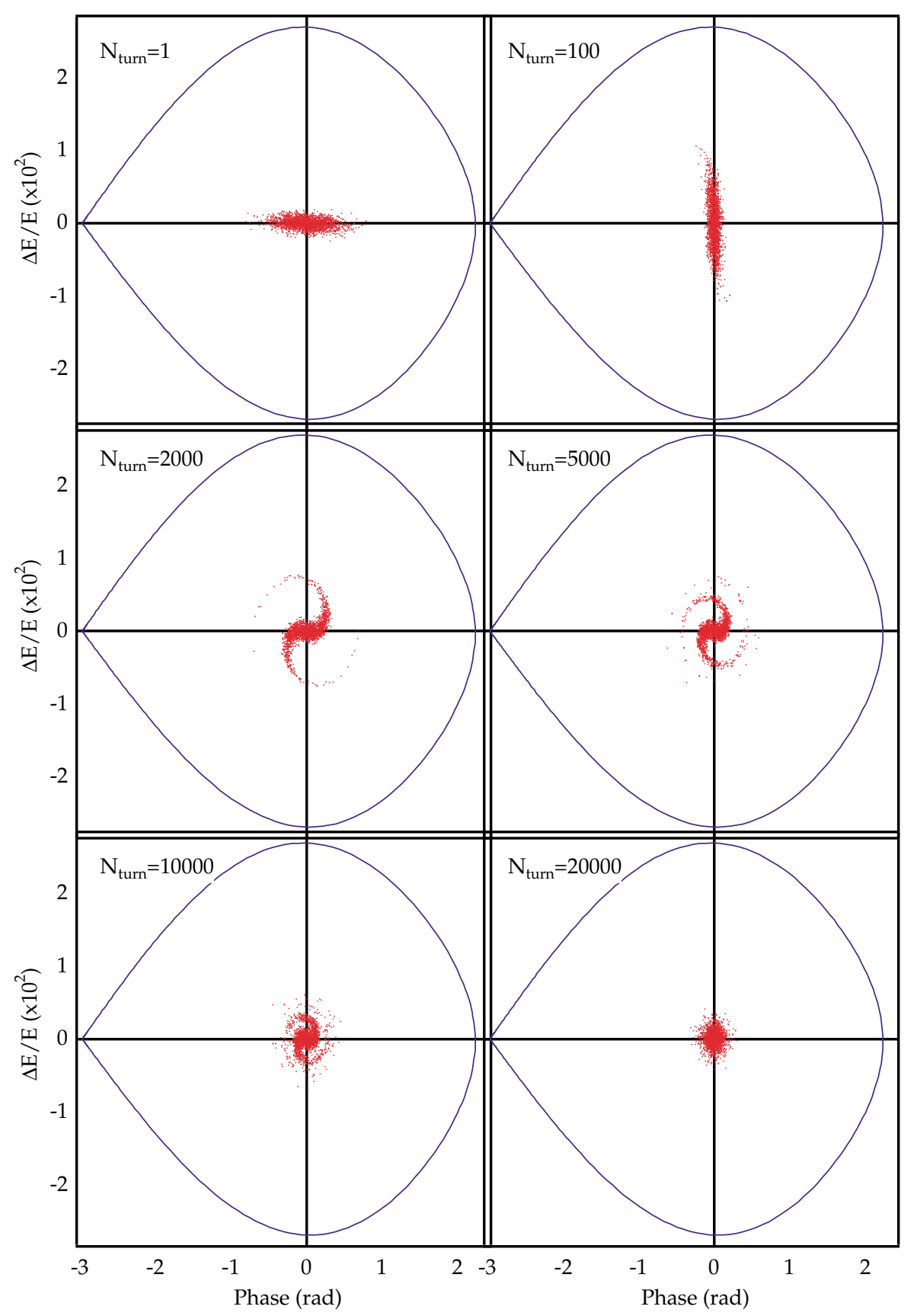

FIG. 2. (Color) Phase space distribution of 5000 particles various times following injection over one radiation damping time. The bunch distribution filaments and then damps to the nominal distribution several damping times later.

(13 msec $\approx \tau)$. As the bunch ellipse rotates in phase space, the projection of the distribution along the phase and energy axes varies with time. In our measurements, we are concerned with projection on the phase axis because that is what we measure with a streak camera. Moshammer has found analytic closed form expressions for the first and second moments of the beam distribution. Below, we summarize the analytic results for the second moment applied to the ALS, where the radiation damping is taken into account. We follow this by a description of the tracking simulation.

\section{A. Analytic formalism}

The time evolution of the second moment of the bunch distribution (i.e., rms bunch length) for an on axis injection is given by

$$
\begin{aligned}
\left\langle z(t)^{2}\right\rangle= & \frac{\sigma_{z^{\infty}}}{\sigma_{\varepsilon^{\infty}}\left[b(t)^{2}+c(t)^{2}\right]} \\
& \times\left\{b(t)-c(t)\left(\operatorname{Re}\left[Z(t)^{3 / 2}\right] \cos \left(2 \omega_{s 0} t\right)\right.\right. \\
& \left.\left.\quad+\operatorname{Im}\left[Z(t)^{3 / 2}\right] \sin \left(2 \omega_{s 0} t\right)\right)\right\}
\end{aligned}
$$


where

$$
Z(t)=\left[1-j \frac{4 f(t) b(t)+f(t)^{2}}{b(t)^{2}-c(t)^{2}}\right]^{-1}
$$

and

$$
f(t)=\frac{\omega_{s 0} \mu \tau}{2} \frac{e^{2 t / \tau}-2\left(u_{0}+v_{0}\right) t / \tau-u_{0} v_{0} e^{-2 t / \tau}-1+u_{0} v_{0}}{e^{2 t / \tau}-u_{0}-v_{0}-u_{0} v_{0} e^{-2 t / \tau}}
$$

where $\omega_{s 0}=2 \pi f_{0} Q_{s}$ is the angular synchrotron frequency at zero oscillation amplitude, $Q_{s}$ is the synchrotron tune, $f_{0}$ is the revolution frequency, and $\tau$ is the longitudinal radiation damping time.

The functions

$$
b(t)=\frac{u(t)+v(t)}{2}
$$

and

$$
c(t)=\frac{u(t)-v(t)}{2}
$$

describe the injected beam longitudinal phase space and depend in turn on the initial conditions through

$$
u(t)=\left[A\left(1-u_{0} e^{-2 t / \tau}\right)\right]^{-1}
$$

and

$$
v(t)=\left[A\left(1-v_{0} e^{-2 t / \tau}\right)\right]^{-1}
$$

where $u_{0}=1-\frac{\sigma_{z 0}^{2}}{\sigma_{z \infty}^{2}}, v_{0}=1-\frac{\sigma_{\varepsilon 0}^{2}}{\sigma_{\varepsilon \infty}^{2}}$, and the final bunch emittance is given by $A=\sigma_{z^{\infty}} \sigma_{\varepsilon^{\infty}}$. The injected bunch length and energy spread are given by $\sigma_{z 0}$ and $\sigma_{\varepsilon 0}$. The natural bunch length and energy spread are given by $\sigma_{z \infty}$ and $\sigma_{\varepsilon^{\infty}}$. The nonlinear rf voltage gives a quadratic dependence of synchrotron tune with amplitude with the coefficient $\mu=\frac{h^{2} \alpha}{8 R Q_{s}}\left[1+\frac{5}{3}\left(\tan \phi_{s}\right)^{2}\right]$, where $h$ is the harmonic number, $R$ is the average radius, and $\phi_{s}$ is the synchronous phase angle.

The bunch length transient obtained from Eq. (1), assuming a radiation damping time $\tau=13.5 \mathrm{~ms}$, is compared to the experimental data in Sec. III.

\section{B. Tracking simulation}

In order to further study the injection transients for a variety of conditions, a simple multiparticle tracking code was developed based on a model by Siemann [11]. The variables used to describe the bunch oscillations are the bunch position $z$, relative to a synchronous electron, and the fractional deviation from the design energy $\varepsilon$. The difference equations used are

$$
\Delta z=-\alpha c T_{0} \varepsilon
$$

and

$$
\begin{aligned}
\Delta \varepsilon= & -2\left(T_{0} / \tau\right) \varepsilon+\frac{V_{\mathrm{rf}}}{E} \cos \left(\phi_{s}+\left(\omega_{\mathrm{rf}} / c\right) z\right) \\
& -\frac{U_{0}}{E}+2 \sigma_{\varepsilon_{\infty}}\left(T_{0} / \tau\right)^{1 / 2} R_{1},
\end{aligned}
$$

where $E$ is the beam energy, $V_{\mathrm{rf}}$ and $\omega_{\mathrm{rf}}$ are the total rf voltage and frequency, $\phi_{s}$ is the synchronous phase angle, $\tau$ is the longitudinal radiation damping time, $\sigma_{\varepsilon^{\infty}}$ is the natural energy spread, and $R_{1}$ is a random Gaussian variable with unit standard deviation. All collective effects are ignored since the injected bunch current is typically $0.25 \mathrm{~mA}$ or less and we assume we are injecting into an empty ring.

In the tracking simulation, initial phase and energy distributions can be set arbitrarily, but we used Gaussian distributions with rms values given by the measured ALS beam parameters at injection. We typically used 5000 particles, but the results are not strongly dependent on this parameter. Tracking results are compared directly with measurements in the following section.

\section{MEASUREMENTS}

To make a detailed study of the longitudinal beam dynamics at injection into the ALS, we used a dual-sweep synchroscan streak camera to observe the evolution of the longitudinal bunch distribution following injection into the storage ring. A schematic diagram of the Hamamatsu C5680 SC is shown in Fig. 3. The SC converts the time structure of a pulse of synchrotron radiation at visible wavelengths from a bend magnet into vertical deflection at the charge-coupled device (CCD) camera. When focused on the photocathode, the transverse spot size of the stored beam is small compared to the vertical sweeping range. In our experiments, the vertical deflection plates are driven by a $125 \mathrm{MHz}$ sinusoidal voltage synchronized to the rf frequency. In addition, there is a slow horizontal deflection which allows observation of the longitudinal profile as a function of time. The time scale of the horizontal sweep can be adjusted to observe a fraction of a turn to several thousands of turns. For horizontal sweep times longer than several hundred turns, individual turns can no longer be resolved and so the longitudinal profile appears as a continuous line across the image. For our measurements we triggered the horizontal sweep of the SC using a signal from the injection system passed through a variable delay in order to sample various times during the injection transient. 


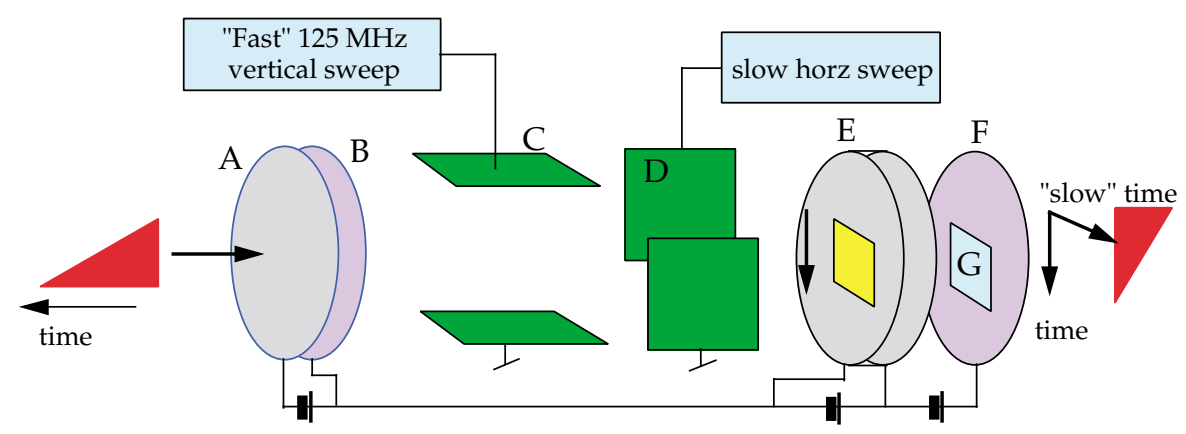

FIG. 3. (Color) Schematic diagram of the streak camera in synchroscan mode with dual sweep. A: photocathode; B: accelerating mesh; C: vertical deflection electrode; D: horizontal deflection electrode; E: microchannel plate; F: phosphor screen; G: CCD camera.

The synchroscan principle is illustrated in Fig. 4 which shows the $125 \mathrm{MHz}$ vertical deflecting voltage with respect to the arrival times of a storage ring bunch. Only deflections within the marked bands appear on the microchannel plate. The phase of the deflecting voltage can be adjusted to place the arrival of a single bunch on the center of the screen. The details of this are further described below for the transient of the uncaptured beam.

The ALS booster can accelerate electrons to a maximum energy of $1.5 \mathrm{GeV}$ with a repetition rate of $1 \mathrm{~Hz}$. Although for normal operations three to four bunches are injected on each booster cycle, for our studies we used only a single bunch with an intensity of about $0.25 \mathrm{~mA}$ per pulse. The energy of the extracted beam can be varied by adjusting the extraction time, requiring retuning of the booster-tostorage ring transfer line. We can also adjust the storage ring energy to match the booster energy by varying either the storage ring dipole field or the rf frequency. For our studies, variation of the rf frequency was the simplest way to compensate for injection energy offsets. To compensate for injection timing or phase offsets, we can adjust the storage ring phase with respect to the injected beam by means of a phase shifter which is in the path of the master oscillator signal to the storage ring rf system. The phase shifter has a range of $300^{\circ}$, allowing us to inject almost all phases in the rf bucket. At maximum energy, the ALS booster has an energy spread almost equal to that of the storage ring and a bunch length about 4 times the storage ring bunch length. It is difficult to vary the booster energy spread, and the bunch length cannot be reduced any further due to limited booster rf power.

\section{A. Injection transient measurements}

In our first measurements, we studied injection offsets of the beam energy and phase. The SC images of the longitudinal beam profile at injection with an energy offset are shown in Fig. 5a. The bunch centroid and length are

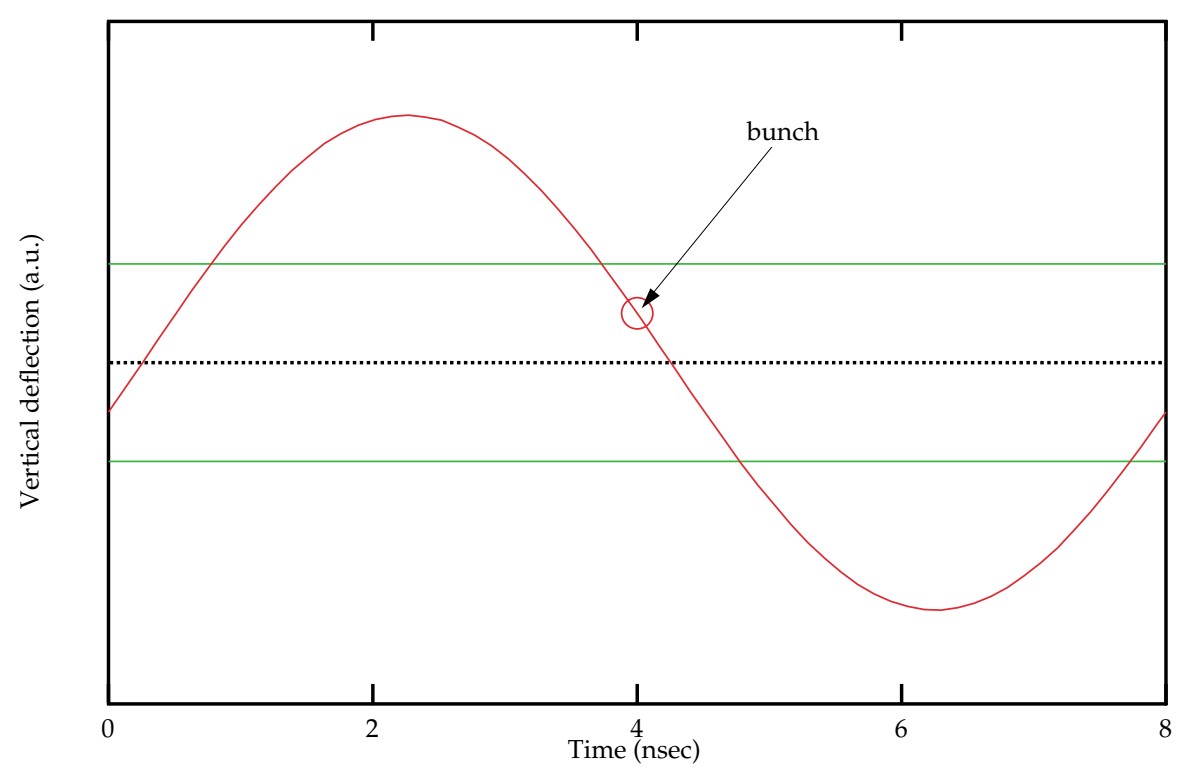

FIG. 4. (Color) Vertical deflecting voltage shown with respect to the arrival times of a storage ring bunch. Only deflections within the marked bands are observed. 


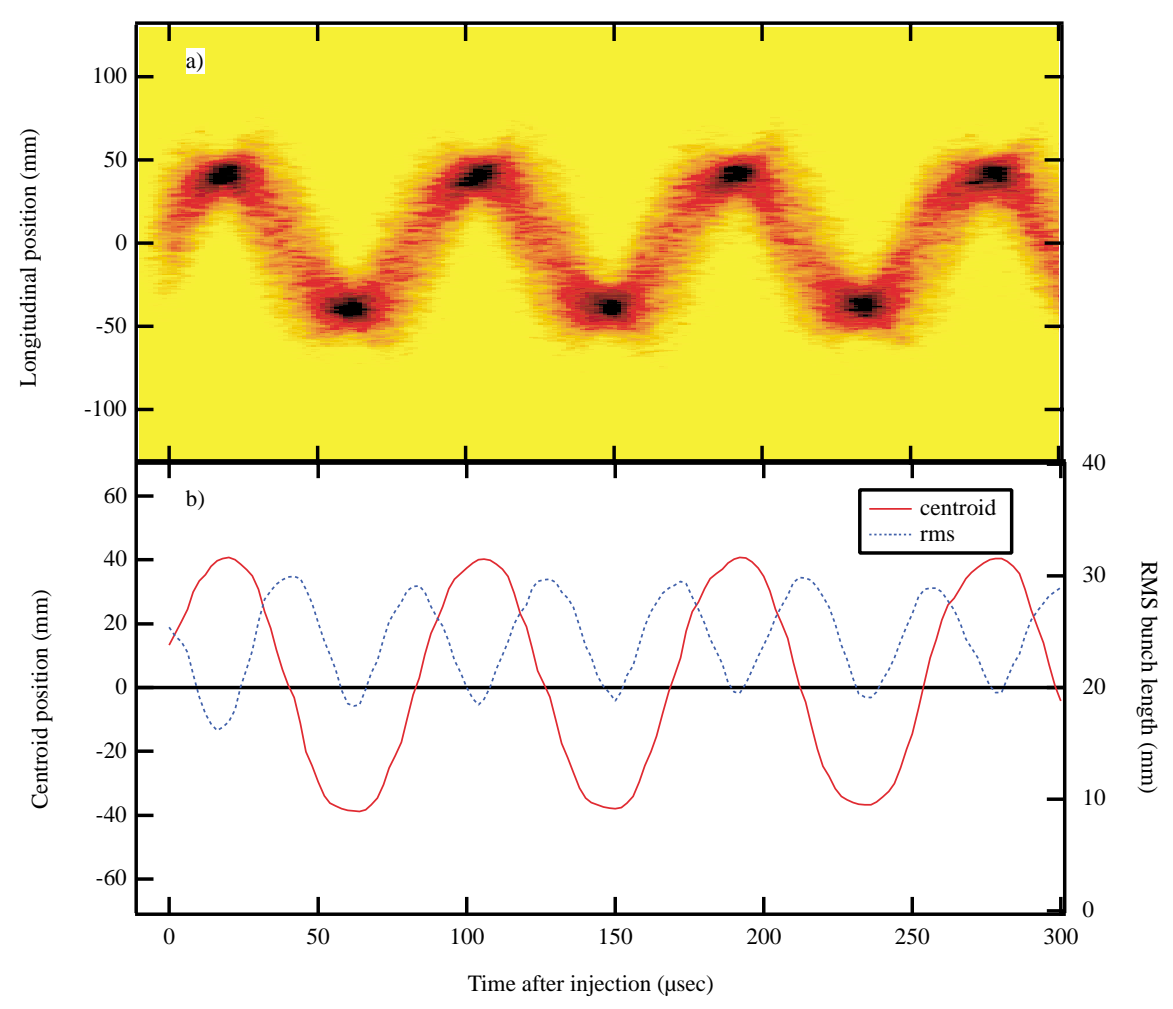

FIG. 5. (Color) (a) Injection transient with an energy offset. (b) Centroid and rms of the bunch distribution.

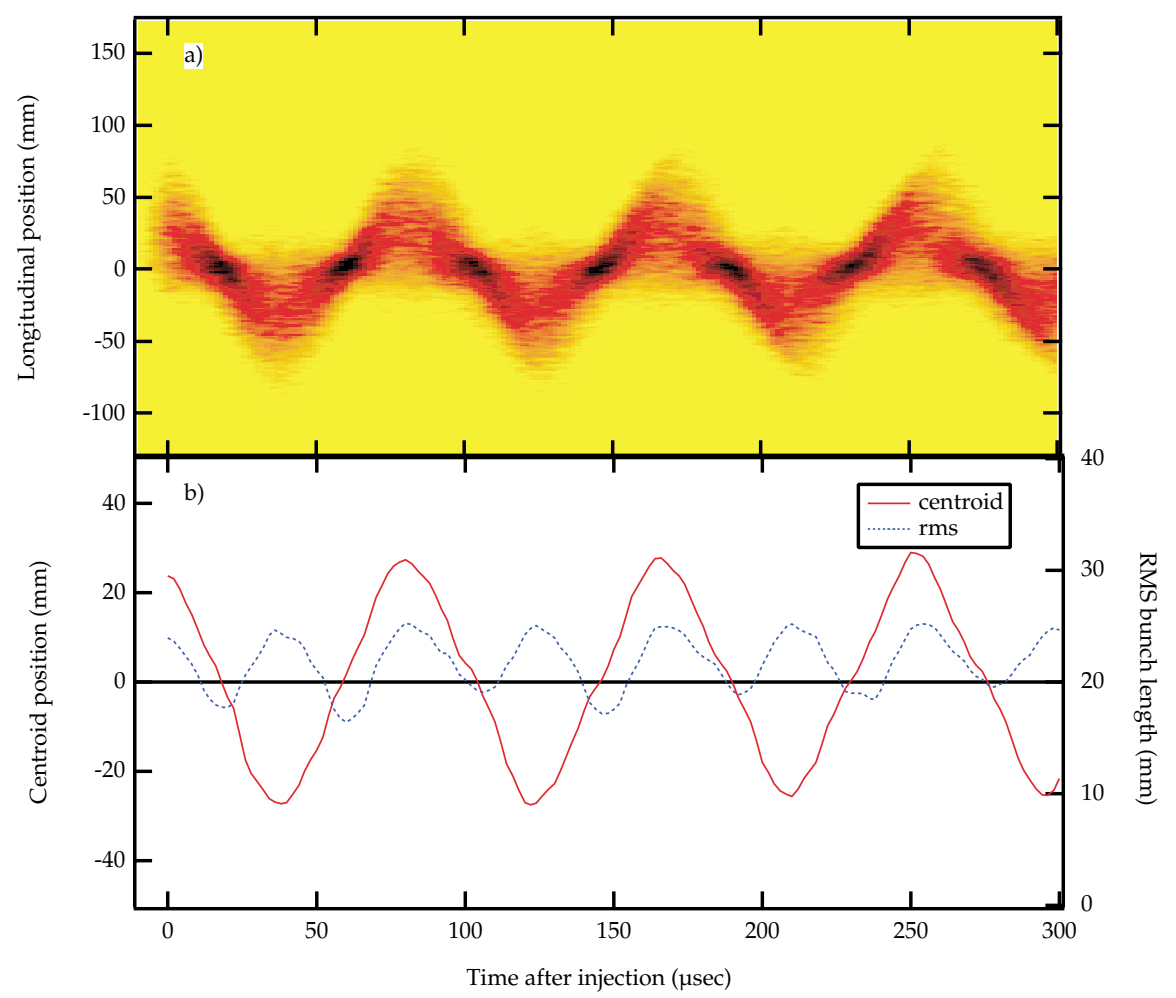

FIG. 6. (Color) (a) Injection transient with a phase offset. (b) Centroid and rms of the bunch distribution. 
shown in Fig. 5b. The SC image and bunch centroid and length for the case of an injected phase offset are shown in Figs. 6a and 6b. The bunch centroid and length are averaged over $5 \mu$ s (7.5 turns) sections of the longitudinal profile. The relative phase of the bunch centroid and shape oscillations can be understood by considering the illustration in Figs. 1a and 1b. For an injected bunch which is elongated in phase, the bunch is expected to be shortest at the zero crossings of a synchrotron oscillation for a phase offset and at the extremes of the oscillation for an energy offset. By observing the relative phase of the bunch length oscillation and the centroid oscillation, we can determine the offset in both energy and phase. To correct the energy and phase offsets, we simply adjust the rf frequency and phase until the centroid oscillation disappears. Although there are other techniques for observing injection offsets, this technique takes advantage of the phase space mismatch to determine whether the offset is energy or phase.

When the phase and energy offsets are corrected, the bunch is injected at the center of the rf bucket with a phase space mismatch as illustrated in Fig. 1c. Two examples of this are shown in Fig. 7. A measurement of the bunch $0.3 \mathrm{~ms}$ following injection is shown in Fig. 7a where the oscillation of the bunch shape is clearly evident but there has been little filamentation. Shown in Fig. 7b is the oscillation $2.7 \mathrm{msec}$ following injection. The filamentation as discussed in Sec. II is evidenced by the apparent curvature in time of the bunch distribution. To compare this result with tracking, we simulated a streak camera picture by combining subsequent phase profiles into an image as shown in Fig. 7c. Using only the measured injection parameters (injected bunch length, energy spread, and synchrotron frequency), the tracking shows good qualitative agreement with the measured result. For a given slice in time, note the presence of "wings" on the longitudinal bunch profile, especially when the bunch is short. The only adjustment made in Fig. 7c is an offset in the horizontal time scale of an amount less than the synchrotron period to align the measured and simulated results. The qualitative agreement is discussed further in this section.

The injected bunch length is determined by measuring the maxima of the bunch length oscillations shown in Fig. 7a. The injected energy spread can be estimated by measuring the longitudinal bunch distribution at the minima of the bunch length oscillations immediately following injection as discussed above. Ignoring decoherence, the
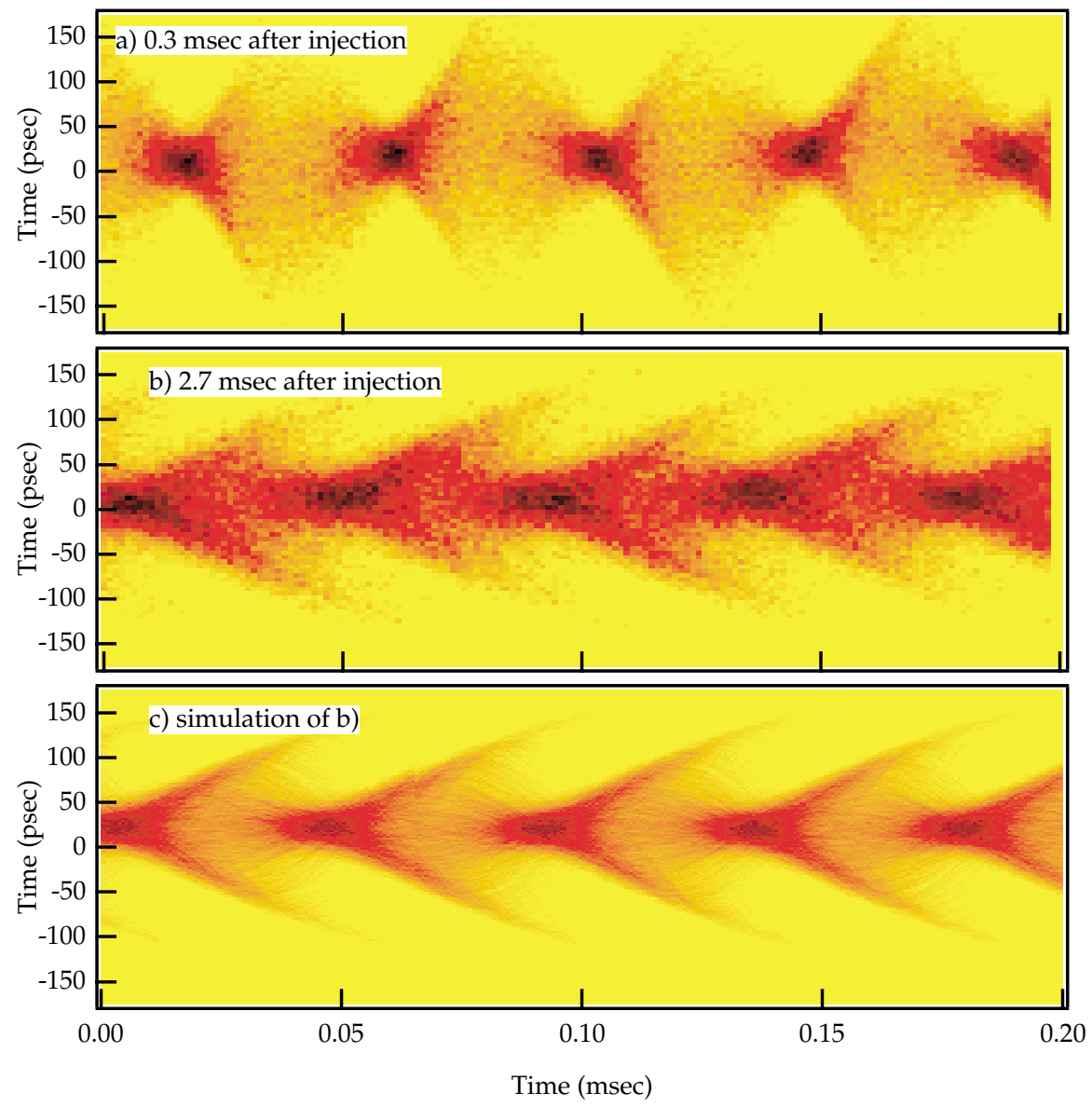

FIG. 7. (a) Measured SC image $0.3 \mathrm{~ms}$ following injection showing modulation of the bunch length. (b) SC image $2.7 \mathrm{~ms}$ after showing filamentation of the large amplitude particles. (c) A computer simulated image found from tracking. 
bunch length at the minima is related to the injected energy spread $\sigma_{\epsilon 0}$ by the relation

$$
\sigma_{z, \min }=\frac{c \alpha}{2 \pi f_{s}} \sigma_{\epsilon 0},
$$

where $f_{s}$ is the synchrotron frequency and $\alpha$ is the momentum compaction. Shown in Fig. 8 is the bunch length at the minima immediately following injection for several settings of the synchrotron frequency, adjusted by varying the rf voltage. From this we arrived at an injected energy spread of $\sigma_{\epsilon 0}=6.5 \times 10^{-4}$.

To study the evolution of the beam over several radiation damping times, it is possible to record the entire injection transient over a maximum of $100 \mathrm{msec}$. However, over this time period, the SC can no longer resolve bunch length oscillations which occur on a time scale of one-half the synchrotron period. To better resolve the oscillations, we recorded millisecond segments of separate transients over the first $35 \mathrm{msec}(\sim 3 \tau)$ following injection. Both phase and energy centroid injection offsets were empirically minimized to less than $10 \%$ of the natural bunch length.

Unfortunately, limitations in the signal level prevented us from accurately measuring the transient oscillations of the bunch during filamentation. We provide here a brief discussion of the limitations since they are typically not present for measurement of a static bunch length in a storage ring. First, the large variability of the bunch length over the first few milliseconds of injection requires a lower adjustment of the SC gain (i.e., multichannel plate gain) such that the signal is not saturated when the bunch is short but is still measurable when the bunch is long. The reduced signal level most strongly affects the accuracy of the bunch length measurement during the filamentation process when the bunch is short. This is because the short rms bunch length is significantly increased by the filamentation wings, as seen in Figs. 7b and 7c. However, for small signal level, it is difficult to resolve the tails. Furthermore, due to the non-Gaussian distribution during filamentation, it is much more difficult to remove the noise using fitting techniques. For example, fitting of the short bunch distribution to a Gaussian always results in a shorter rms bunch length than predicted because the fitting tends to ignore the wings. However, it is much easier to measure the bunch when it is longer because the bunch length is mostly determined by the core of the distribution and not the wings. The signal/noise in the wings can be improved by averaging over multiple injection pulses. However, shot-to-shot jitter of either the energy or timing of the pulses will begin to blur the results. We empirically found that a maximum of five averages was useful. The net result of our analysis is that we cannot simultaneously measure the short and long bunch lengths during the filamentation, and thus, in this paper, we can only accurately report the peak of the bunch length oscillations and compare them with theory and simulation.

The measured peak bunch length over the entire transient is shown in Fig. 9a. The transient calculated from Eq. (1) using the measured injection conditions is overlaid on the measured transient. The transient obtained from tracking using the measured injection beam parameters is shown in Fig. 9b. The peak amplitude of the observed transient is in very good agreement with the calculated and simulated transients. Note that, because of the particular ALS injection parameters, there is no time where the bunch length decays exponentially with the radiation damping time, making it difficult to directly measure. The effective decay rate is about half the radiation damping time.

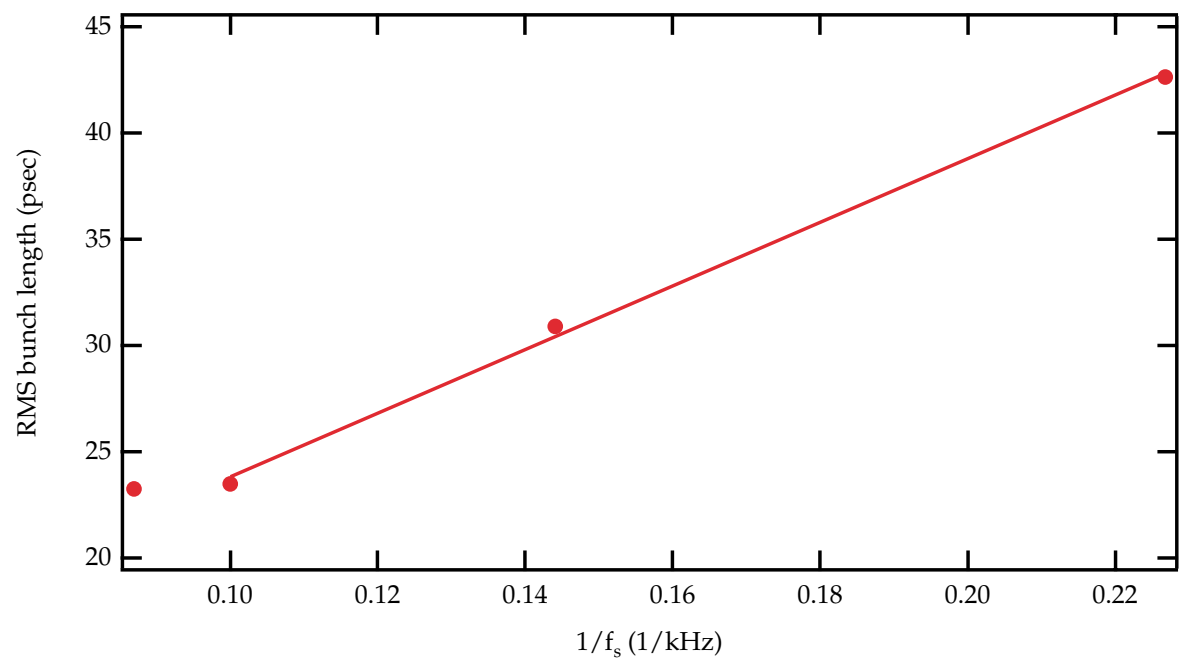

FIG. 8. (Color) rms bunch length at minima of quadrupole oscillations vs synchrotron frequency. The slope is proportional to the injected energy spread of the bunch. 


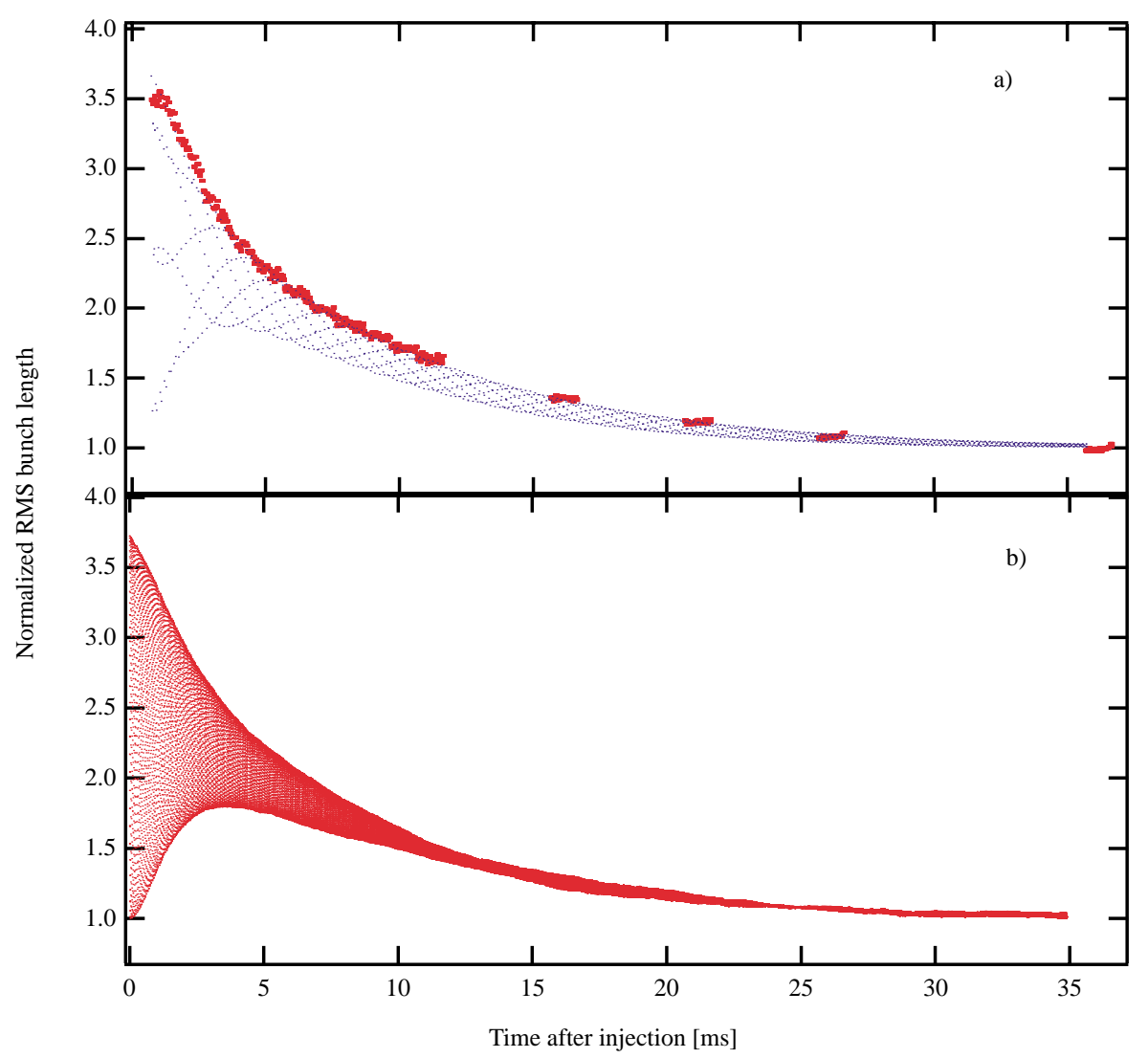

FIG. 9. (Color) (a) Measured peak rms bunch length over an entire injection cycle. The first 11 msec consist of 1 msec segments. The calculated bunch length using measured injection parameters is also shown. (b) Transient from numerical tracking.

\section{B. Decaying beam transients}

During our studies of longitudinal injection transients described above, we had occasion to observe the decay of the beam with the rf system turned off. This is always an undesirable situation since it is impossible to store beam for longer than a few tens of milliseconds. However, we realized the application of this observation as a technique to measure the energy loss per turn due to synchrotron radiation and potentially some other interesting machine parameters. Although it is possible to measure the energy loss per turn in other ways, we believe this technique is the most direct and accurate, given the availability of a dual sweep SC. We describe this technique below and give an example of its application at the ALS.

When an electron bunch is injected into the storage ring, it begins to lose energy to synchrotron radiation. Its orbit spirals inward and the beam eventually hits an aperture and is lost. The ring orbit period also varies according to the relation

$$
\frac{\Delta T}{T_{0}}=\alpha \delta_{\mathrm{tot}}
$$

where $\alpha$ is the momentum compaction and $\delta_{\text {tot }}$ is the total fractional energy difference of the beam from the injected energy. Above transition, we expect the orbit period to decrease with time for a decrease in beam energy $(\alpha>0$, $\delta_{\text {tot }}<0$ ). Immediately following injection, we can assume the energy loss per turn is constant and $\alpha$ is independent of energy. In this case, the total path difference (in units of time) can be expressed as

$$
\Delta T_{\mathrm{tot}}=\frac{1}{2} \frac{f_{0}}{E} \alpha U_{0} t^{2},
$$

where $t$ is the time following injection. The quadratic dependence of the path difference on time results from integrating the linear energy difference per turn.

As the bunch arrives earlier each turn, we will observe an increasing vertical deflection on the SC as seen in Fig. 4 until the path length difference is larger than a quarter wavelength of the synchroscan frequency whereupon the vertical deflection will decrease. This is illustrated in Fig. 10.

Figure 11a shows a SC image at injection with the storage ring rf turned off. An expanded view of the first $100 \mu \mathrm{s}$ is shown in Fig. 11b. Note that Fig. 11a shows 


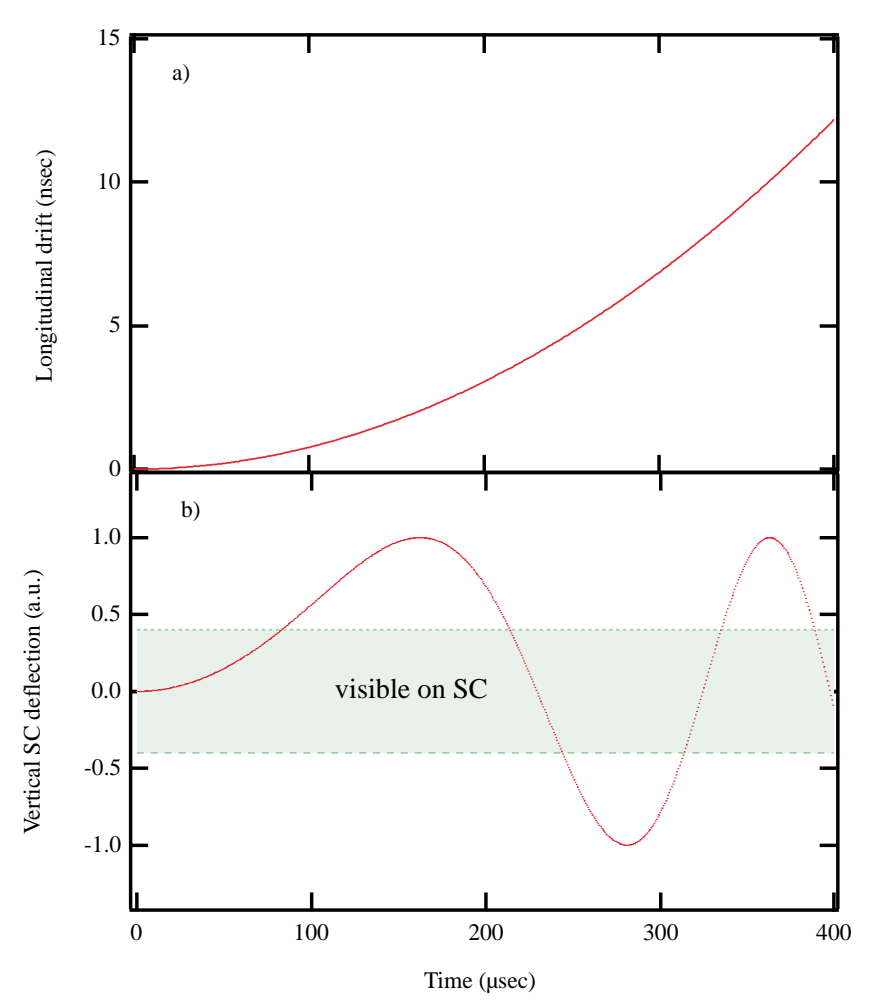

FIG. 10. (Color) (a) Longitudinal drift of a bunch injected with no rf. (b) Corresponding vertical SC deflection for the drift shown in (a).

the characteristic expected in Fig 10. A fit of the orbit period difference using Eq. (12) is also shown in Fig. 11b. This gives a value for the radiation loss per turn as $94 \mathrm{keV}$, close to the expected value of $92 \mathrm{keV}$. It may be possible to derive more information from the evolution of the bunch length. We are currently analyzing this.

\section{CONCLUSIONS}

We have presented experimental observations of injection transients in longitudinal phase space using a dual-scan synchroscan streak camera in an electron storage ring. From these measurements, we have observed and corrected phase and energy offsets at injection, determined the injected bunch length and energy spread, directly observed phase space filamentation, and recorded the evolution of the peak bunch length over several radiation damping times following injection. This is clearly a valuable technique for understanding injection in longitudinal phase space and will be useful in studying longitudinal aspects of continuous top-up injection in the ALS. We have also demonstrated the novel technique of measuring the transient decay of an uncaptured beam that may be useful as an independent means of measuring the energy loss per turn.
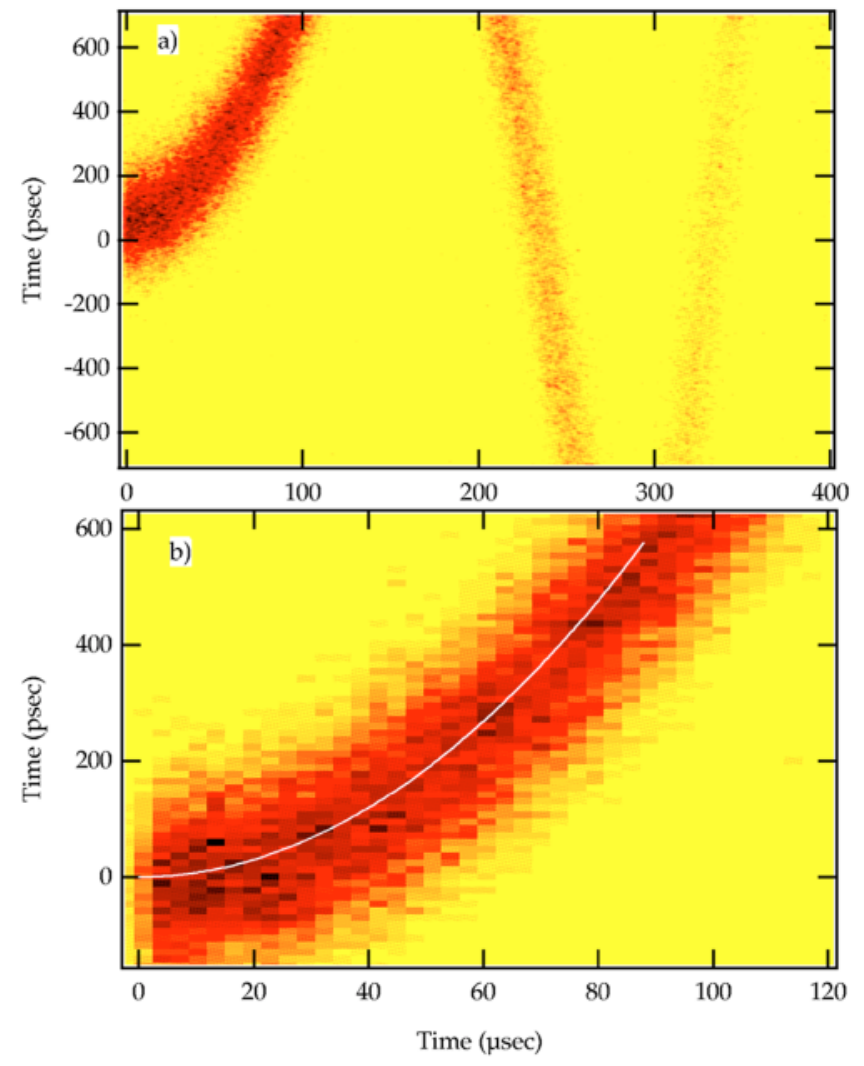

FIG. 11. (Color) Transient of a decaying, uncaptured electron beam at injection. A fit to the drift is shown as a white line.

\section{ACKNOWLEDGMENTS}

We would like to thank Chris Timossi and Michael Fahmie for providing the streak camera pretrigger. We would also like to acknowledge several useful discussions with Albert Hofmann and David Robin. This work was supported by the U.S. Department of Energy under Contract No. DE-AC03-76SF00098

[1] R. E. Meller, A. W. Chao, J. M. Peterson, S. G. Peggs, and M. Furman, Superconducting Supercollider Report No. SSC-N 360, 1987 (unpublished).

[2] A. Chao et al., Phys. Rev. Lett. 61, 2752 (1988).

[3] S. Y. Lee et al., Phys. Rev. Lett. 67, 3768 (1991).

[4] D. Caussyn et al., Phys. Rev. A 46, 7942 (1992).

[5] J. M. Byrd and D. Sagan, in Proceedings of the 1991 Particle Accelerator Conference, San Francisco (IEEE, New York, 1991), p. 1080.

[6] H. Moshammer, Phys. Rev. E 48, 1390 (1993).

[7] H. Moshammer, Nucl. Instrum. Methods Phys. Res., Sect. A 323, 553 (1992).

[8] H. Moshammer, Phys. Rev. E 48, 2140 (1993).

[9] C. Simopoulos and R. L. Holtzapple, in Proceedings of the 1995 Particle Accelerator Conference, Dallas, 1995 (IEEE, Piscataway, NJ, 1996). 
[10] R. L. Holtzapple, R. H. Siemann, and C. Simopoulos, in Proceedings of the 1995 Particle Accelerator Conference, Dallas, 1995 (Ref. [9]).

[11] R. Siemann, in Physics of High Energy Particle Accelera- tors, edited by Melvin Month, Per F. Dahl, and Margaret Dienes, AIP Conf. Proc. No. 127 (AIP, New York, 1983), p. 431. 